https://doi.org/10.35336/VA-2021-2-5-10

ОПЫТ ПРИМЕНЕНИЯ МОДУЛЯЦИИ СЕРДЕЧНОЙ СОКРАТИМОСТИ У ПОЖИЛЫХ ПАЦИЕНТОВ

С СЕРДЕЧНОЙ НЕДОСТАТОЧНОСТЬЮ СО СНИЖЕННОЙ ФРАКЦИЕЙ ВЫБРОСА ЛЕВОГО

ЖЕЛУДОЧКА: РЕЗУЛЬТАТЫ 12-МЕСЯЧНОГО НАБЛЮДЕНИЯ

И.А.Чугунов ${ }^{1}$, К.В.Давтян ${ }^{1}$, А.Г.Топчян ${ }^{1}$, Н.А.Миронова ${ }^{2}$, Е.М.Гупало ${ }^{2}$

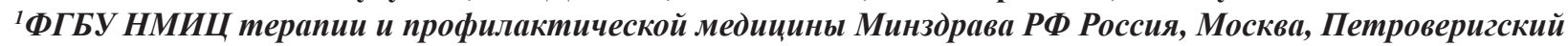
переулок 10, с. 3; 'ФГБУ НМИЦ Кардиологии Минздрава РФ Россия, Москва, 3-я Черепковская 13 A

Цель. Оценить эффективность и безопасность применения модуляции сердечной сократимости (МСC) для лечения пациентов старше 65 лет с хронической сердечной недостаточностью с низкой фракцией выброса (СНнФВ).

Материал и методы. В исследование были включены 16 пациентов с СНнФВ и II-IV функциональным классом (II - 9 (56\%) пациентов, III - 4 (25\%), IV - 3 (19\%)) по NYHA старше 65 лет (медиана возраста 70 лет) на оптимальной медикаментозной терапии $\mathrm{CH}$, которым были имплантированы системы MCC: устройство Оптимайзер (Impulse Dynamics) и два правожелудочковых электрода. Перед включением в исследование пациентам проводились тест с шестиминутной ходьбой (ТШХ), трансторакальная эхокардиография (ЭхоКГ) и анализ крови на NTproBNP. Срок наблюдения составил 12 месяцев с контрольными визитами через 2, 6 и 12 месяцев после имплантации. Контрольные ЭхоКГ, ТШХ и анализ крови на NTproBNP повторно выполнялись во время плановых визитов через 6 и 12 месяцев.

Результаты. Из 16 пациентов, включенных в исследование 2 умерли на 158 и 243 день наблюдения от декомпенсации СН. Пациенты, прошедшие 12-ти месячный период наблюдения, показали значимое увеличение дистанции шестиминутной ходьбы (350 метров до, 402,5 метра после, $\mathrm{p}=0,01)$ и тенденцию к увеличению ФВ левого желудочка (33\% до, 40\% после p=0,2) и снижению NTproBNP (1112 пг/мл до 527 пг/мл после, $\mathrm{p}=0,19)$.

Заключение. Применение МСС-терапии у пациентов со СНнФВ старше 65 лет является безопасным и эффективным методом в качестве дополнительной опции к оптимальной медикаментозной терапии для снижения выраженности симптомов СН.

Ключевые слова: модуляция сердечной сократимости; сердечная недостаточность; имплантируемые устройства; пожилые пациенты

Конфликт интересов: не заявляется

Рукопись получена: 27.01.2021 Исправленная версия получена: 25.05.2021 Принята к публикации: 27.05.2021 Ответственный за переписку: Иван Александрович Чугунов, E-mail: vvizevvolf@gmail.com

Данная научная работа представлена в рамках конкурса молодых ученых IX Всероссийского съезда аритмологов, проводимого 20-22 мая 2021 года в Санкт-Петербурге

И.А.Чугунов - ORCID ID 0000-0001-7077-9644, К.В.Давтян - ORCID ID 0000-0003-3788-3997, А.Г.Топчян - ORCID ID 0000-0001-7605-6316, Н.А.Миронова - ORCID ID 0000-0002-2374-3718, Е.М.Гупало - ORCID ID 0000-0002-0063-5474

Для цитирования: Чугунов ИА, Давтян КВ, Топчян АГ, Миронова НА, Гупало ЕМ. Опыт применения модуляции сердечной сократимости у пожилых пациентов с сердечной недостаточностью со сниженной фракцией выброса левого желудочка: результаты 12-месячного наблюдения. Вестник аритмологии. 2021;28(2): 5-10. https://doi.org/10.35336/VA-2021-2-5-10.

\title{
CARDIAC CONTRACTILITY MODULATION THERAPY IN ELDERLY PATIENTS WITH CHRONIC HEART FAILURE: RESULTS OF THE 12-MONTH FOLLOW-UP \\ I.A.Chugunov $^{1}$, K.V.Davtyan ${ }^{1}$, A.H.Topchyan ${ }^{1}$, N.A.Mironova ${ }^{2}$, E.M.Gupalo ${ }^{2}$
}

${ }^{1}$ National Medical Research Center for Therapy and Preventive Medicine, Russian Federation, Moscow, Petroverigsky lane 10 c. 3; ${ }^{2}$ National Medical Research Center of Cardiology, Russian Federation, Moscow, 3rd Cherepkovskaya 13 A

Aim. This study aimed to evaluate the efficacy and safety of cardiac contractility modulation (CCM) therapy in elderly patients with heart failure with reduced ejection fraction (HFrEF).

Methods. Sixteen patients older than 65 years old (median age 70 years) undergoing CCM Optimizer (Impulse Dynamics) device implantation due to HFrEF (NYHA class II - 9 (56\%), III - 4 (25\%), IV - $3(19 \%)$ ) were enrolled in this two-center observational study. Before implantation 6-minute walk test (6MWT), transthoracic echocardiography (TTE) was performed on all patients, and NTproBNP levels were assessed. The follow-up duration was 12 months with 2, 6, 12-month follow-up visits. Control 6MWT, TTE and NTproBNP tests were performed at 6-month and 12-month follow-up visits.

Results. Two patients died during follow-up due to HF decompensation. The remaining patients showed a significant improvement in $6 \mathrm{MWT}$ ( $350 \mathrm{~m}$ vs $402.5 \mathrm{~m}, \mathrm{p}=0,01)$. We also noted a tendency towards the left ventricular EF improvement ( $33 \%$ vs $40 \%, \mathrm{p}=0,2)$ and lower values of NTproBNP levels $(1112 \mathrm{pg} / \mathrm{ml}$ vs $527 \mathrm{pg} / \mathrm{ml}, \mathrm{p}=0,19)$. 
Conclusion. CCM therapy is a safe and efficient additional treatment option to manage elderly patients with HFrEF for reducing signs and symptoms of HF.

Keywords: cardiac contractility modulation; heart failure; device-based therapy; elderly

Conflict of Interests: nothing to declare

Received: 27.01.2021 Revision received: 25.05.2021 Accepted: 27.05.2021

Corresponding author: Chugunov Ivan, E-mail: vvizevvolf@gmail.com

This work was presented as part of the competition for young scientists of the IX All-Russian Congress of Arrhythmologists, held on May 20-22, 2021 in St. Petersburg

I.A.Chugunov - ORCID ID 0000-0001-7077-9644, K.V.Davtyan - ORCID ID 0000-0003-3788-3997, A.H.Topchyan ORCID ID 0000-0001-7605-6316, N.A.Mironova - ORCID ID 0000-0002-2374-3718, E.M.Gupalo - ORCID ID 00000002-0063-5474

For citation: Chugunov IA, Davtyan KV, Topchyan AH, Mironova NA, Gupalo EM. Cardiac contractility modulation therapy in elderly patients with chronic heart failure: results of the 12-month follow-up. Journal of Arrhythmology. 2021;28(2): 5-10. https://doi.org/10.35336/VA-2021-2-5-10.

Модуляция сердечной сократимости (МСС) новый дополнительный метод лечения хронической сердечной недостаточности (ХCH). МСС терапия нормализует экспрессию белков саркоплазматического ретикулума, в частности SERCA-2а - белка, контролирующего транспорт ионов кальция в саркоплазматический ретикулум во время диастолы. Также экспериментальные данные указывают на положительную роль МСC терапии в процессе обратного ремоделирования миокарда $[1,2]$. Внешне система состоит из генератора импульсов (Оптимайзер) и двух правожелудочковых электродов, терапия наносит высокоамплитудные стимулы на межжелудочковую перегородку в течение абсолютного рефрактерного периода кардиомиоцита [3].

Доступные клинические данные подтверждают положительное влияние МСС-терапии у пациентов с симптомной $\mathrm{CH}$, получающих оптимальную медикаментозную терапию: этот тип терапии показал увеличение фракции выброса левого желудочка (ФВЛЖ), дистанции шестиминутной ходьбы и качества жизни $[4,5]$. Кроме того, по результатам клинических исследований снижение сердечно-сосудистой смертности и количества госпитализаций, связанных с $\mathrm{CH}$, также отмечается у пациентов с ФВЛЖ 35-45\% [6]. Однако данные об использовании МСС у пожилых ограничены и представлены единичными описаниями клинических

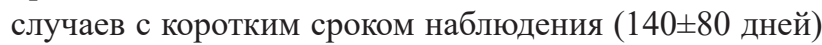
пациентов, которые не ответили на сердечную ресинхронизирующую терапию (СРТ) [7].

Целью нашего исследования было оценить эффективность и безопасность применения МСС-терапии у пациентов старше 65 лет в дополнение к оптимальной фармакотерапии сердечной недостаточности.

\section{МАТЕРИАЛ И МЕТОДЫ ИССЛЕДОВАНИЯ}

\section{Клиническая характеристика пациентов}

В исследование были включены 16 пациентов с низкой ФВЛЖ $(<40 \%)$ и II-IV функциональным классом (ФК) по NYHA, которым в двух клиниках в период с 2016 по 2018 гг были имплантированы устрой- ства MCC (Optimizer IV или Optimizer smart, Impulse Dynamics). Медиана возраста пациентов составила 70 (66-72) лет, 14 из них (87,5\%) были мужчины. У тринадцати пациентов $(81,25 \%)$ причиной развития $\mathrm{CH}$ была ишемическая болезнь сердца (ИБС), у одного пациента - дилатационная кардиомиопатия (ДКМП). Еще один пациент страдал саркоидозом с поражением сердца. На момент имплантации устройства МСС пятеро пациентов $(31,25 \%)$ имели ранее имплантированный кардиовертер-дефибриллятор (ИКД) или СРТ с функцией дефибриллятора в рамках профилактики внезапной сердечной смерти (BCC). Пациенты получали максимально переносимую медикаментозную терапию CH: ингибиторы ангиотензин превращающего фермента / антагонисты рецепторов ангиотензина, бетаадреноблокаторы и антагонисты минералокортикоидов, согласно действующим рекомендациям, оптимальную дозировку препаратов не удавалось назначить в большинстве случаев из-за гипотонии. Пациенты с ИБС получили максимальную коронарную реваскуляризацию. Характеристики пациентов приведены в табл. 1.

\section{Процедура имплантации устройств MCC-mepanuu}

Имплантация устройства выполнялась под местной анестезией в правой подключичной области. Через правую подключичную вену и, при возможности, через правую плечеголовную вену в сердце проводились электроды: для устройств Optimizer IV - два желудочковых и один предсердный электрод, для устройств Optimizer Smart - два желудочковых электрода. Желудочковые электроды (далее маркируемые как local sense - локальная чувствительность (LS) и right ventricle (RV) - правожелудочковый), имплантировались в межжелудочковую перегородку с диастазом не менее 2 см. Выполнялись стандартные тесты чувствительности и порога стимуляции с соблюдением следующих требований: чувствительность не менее 5 мВ и порог стимуляции менее 1,5 В. При необходимости (имплантация электрода близко к верхушке ПЖ или передней стенке ПЖ) проводилась проверка стимуляции диафрагмального нерва при стимуляции с каждого электрода с ам- 
плитудой 10 В. При имплантации Optimizer IV предсердный электрод имплантировался в область ушка правого предсердия, или при неудовлетворительных параметрах - в боковую стенку правого предсердия.

MCC-генератор подключался к электродам, после чего выполнялась настройка устройства с помощью наружного программатора. Интраоперационная настройка была необходима для своевременного выявления случаев неудовлетворительной морфологии желудочкового сигнала на LS канале, а также плохой субъективной переносимости терапии пациентом. Желудочковый сигнал на LS электроде должен был быть высокоамплитудным, чтобы исключить ошибки по типу двойного восприятия.

Несколько пациентов имели значительный фиброз межжелудочковой перегородки, в результате чего регистрировался фрагментированный желудочковый сигнал. Демонстрация экрана с нормальной

Таблица 1.

Характеристика пациентов

\begin{tabular}{|c|c|}
\hline Показатели & Значения \\
\hline Всего пациентов, $\mathrm{n}$ & 16 \\
\hline Возраст, лет & $70(66-72)$ \\
\hline Мужчины, n (\%) & $14(87,5 \%)$ \\
\hline ИБС, n $(\%)$ & $14(87,5 \%)$ \\
\hline Артериальная гипертензия, n (\%) & $15(93,75 \%)$ \\
\hline Фибрилляция предсердий, n (\%) & $13(81,25 \%)$ \\
\hline Сахарный диабет, n (\%) & $3(18,75 \%)$ \\
\hline Наличие ИКД/СРТ-Д*, n (\%) & $5(31,25 \%)$ \\
\hline ХБП ІІІ стадии и тяжелее, n (\%) & $4(25 \%)$ \\
\hline II ФК по NYHA, n & 9 \\
\hline III ФК по NYHA, n & 4 \\
\hline IV ФК по NYHA, n & 3 \\
\hline Индекс массы тела, кг/м² (медиана, ИКР) & $27,3[24,0 ; 29,9]$ \\
\hline ФВ ЛЖ, \% (медиана, ИКР) & $34[31 ; 38]$ \\
\hline Средняя дистанция ТШХ, м (медиана, ИКР) & $340[200 ; 390]$ \\
\hline NTproBNP пг/мл (медиана, ИКР) & $1007[199 ; 2241]$ \\
\hline \multicolumn{2}{|l|}{ Получаемая терапия } \\
\hline Ингибиторы АПФ, n (\%) & $11(68,75 \%)$ \\
\hline Антагонисты рецепторов ангиотензина, n (\%) & $2(12,5 \%)$ \\
\hline Ингибиторы рецепторов неприлизина, n (\%) & $3(18,75 \%)$ \\
\hline Бета-блокаторы, n (\%) & $13(81,25 \%)$ \\
\hline Антагонисты минералокортикоидов, n (\%) & $16(100 \%)$ \\
\hline Дигоксин, n (\%) & $3(18,75 \%)$ \\
\hline Амиодарон, n (\%) & $3(18,75 \%)$ \\
\hline
\end{tabular}

Примечание: ИБС - ишемическая болезнь сердца, ИКД - имплантируемый кардиовертер-дефибриллятор, СРТ-Д - устройство сердечной ресинхронизирующей терапии с функцией дефибриллятоpa, * - на момент имплантации устройства модуляции сердечной сократимости, ХБП - хроническая болезнь почек, ФК - функциональный класс, ИКР - интерквартильный размах, ФВ ЛЖ - фракция выброса левого желудочка, ТШХ - тест с шестиминутной ходьбой, АПФ - ангиотензин превращающий фермент (а) и фрагментированной (б) морфологиями приведены на рис. 1.

Регистрация такого сигнала требует репозиции электродов до получения удовлетворительной морфологии сигнала. Однако у одного из пациентов фиброз межжелудочковой перегородки был настолько распространен, что при неоднократной репозиции электрода добиться монофазного сигнала не удалось. В случае, когда репозиция не решила проблему, устройство было перепрограммировано таким образом, чтобы ранний сигнал оказался в слепом периоде до начала окна “Alert” (рис. 2). В остром и отсроченном периоде у данного пациента удалось достичь более $80 \%$ эффективной стимуляции и клинического эффекта МСС-терапии.

Отдельной проблемой является присутствие преходящей внутрижелудочковой блокады. При настройке системы Optimizer можно наблюдать преходящие изменения временных интервалов между сигналами на RV и LS каналах. У пациентов с подобными нарушениями проводимости и имплантированным Optimizer-ом наблюдается значительное снижение общего времени наносимой терапии, поскольку прибор не обладает алгоритмами для подобной ситуации.

Имплантация устройств СРТ пациенту, у которого уже имеется Optimizer, встречает аналогичную сложность настройки, поскольку возбуждение к электродам приходит сразу с нескольких направлений. Оптимальное программирование ресинхронизирующей терапии, как метода с высшим уровнем рекомендаций, находится в приоритете, согласно действующим рекомендациям. Тем не менее, имплантация МСС и СРТ приборов в данном наблюдении к конфликтам не привела.

После настройки оптимальных параметров включалась МСС-терапия. При появлении непереносимости/субъективного дискомфорта в момент включения терапии проводилась репозиция симптом-связанного электрода. Во избежание стимуляции ложа изза повреждения изоляции электродов, устройство помещалось в отдельное от электродов ложе. При наличии имплантированного ранее ИКД выполнялась проверка на перекрестную чувствительность работы двух устройств. В остальном имплантация выполнялась аналогично имплантации ЭКС. Время пребывания в стационаре после имплантации составляло от 4 до 7 дней.

У одного пациента интраоперационно развился синус-арест, что потребовало постановку временного кардиостимулятора. У пациента в анамнезе отсутствовали данные, указывающих на наличие синдрома слабости синусового узла, пациент на временной кардиостимуляции был переведен в отделение интенсивной терапии для динамического наблюдения. Синус-арест разрешился на следующие сутки и в дальнейшем не реци- 
дивировал. Данному пациенту в дальнейшем был имплантирован ИКД, который при проверках не обнаружил потребности пациента в стимуляции.

Важность интраоперационной настройки устройства подчеркивает опыт одного из пациентов, которому через день после имплантации была выполнена репозиция желудочкового электрода в связи с появлением жалоб на пульсирующий дискомфорт в грудной клетке, связанный с моментом включения MCC-терапии. Пациент имел тяжелую $\mathrm{CH}$ на фоне максимально подобранной терапии (до ортопное), положение лежа на операции переносил крайне плохо. В процессе тестирования терапии у пациента развилась острая левожелудочковая недостаточность, в связи с чем переносимость терапии оценить не удалось. Симптомность стимуляции стала очевидной только на вторые сутки после операции, когда пациент смог оценить собственное самочувствие. Пациенту была выполнена репозиция электрода, после чего жалобы больше не беспокоили.

Срок наблюдения пациентов составил 12 месяцев с графиком плановых визитов через 2, 6 и 12 месяцев после имплантации МСС. На каждом визите проводились сбор анамнеза, осмотр и при необходимости коррекция терапии. На 6-м и 12-м месячных визитах также проводились ТШХ, анализ крови, определение уровня NTproBNP в крови и трансторакальная эхокардиография (ЭхоКГ). Кроме того, контролировалась работа модулятора сердечной сократимости, проводилась коррекция работы устройств для достижения максимального процента работы.

В течение 12-месячного наблюдения по данным выполненных ЭхоКГ проводилась повторная стратификация риска ВСС, 4 пациентам был имплантирован ИКД ввиду сохранения / снижения ФВ ЛЖ ниже $35 \%$. Пяти пациентам ИКД не был имплантирован по причине повышения ФВ выше 35\% и отсутствия показаний для вторичной профилактики ВСС. Кроме того, одна пациентка, не дожила до имплантации, еще одному пациенту ИКД не был имплантирован по социальным причинам.

\section{Статистический анализ}

Статистический анализ выполнялся с использованием программы Stata (v15.0 для Windows, StataCorp., США). Для описания количественных переменных применялись медиана и интерквартильный размах, качественные переменные описывались абсолютными и относительными частотами (процентами). Для сравнения зависимых выборок применялся тест Краскелла-Уоллиса. При сравнении качественных показателей использовался $\chi^{2}$-критерий Пирсона и точный тест Фишера. Различия считались статистически достоверными при значениях двухстороннего $\mathrm{p}<0,05$.

\section{ПОЛУЧЕННЫЕ РЕЗУЛЬТАТЫ}

Годовая сердечно-сосудистая смертность составила 13,3\%: двое пациентов умерли на 158 и 243 дни наблюдения по причине декомпенсации СН. Оба этих пациента имели ИБС и перенесенный инфаркт миокарда в анамнезе. Исходно одна пациентка имела II ФК по NYHA и ФВ ЛЖ 34\%, второй пациент имел ИКД (в рамках вторичной профилактики), IV ФК по NYHA, ФВ ЛЖ $38 \%$ и тяжелую митральную недостаточность, срабатываний устройства у него не отмечалось. Данному пациенту была предложена имплантация устройства как терапия отчаяния ввиду отсутствия достаточного эффекта от максимально возможной для него терапии.

По данным 12-месячного наблюдения у пациентов отмечалось улучшение переносимости физических нагрузок - значимо увеличилась дистанция шестиминутной ходьбы $(\mathrm{p}=0,0079)$, имелась тенденция к уменьшению функционального класса сердечной недостаточности по классификации NYHA. Также регистрировалась тенденция к снижению показателя NTproBNP и повышению ФВЛЖ, однако эти изменения не достигли статистически значимого уровня. При оценке стандартных ЭхоКГ параметров также наблюдалась общая тенденция к уменьшению размеров и объема левого желудочка. Тем не менее эта тенденция не достигла статистической значимости (табл. 2).

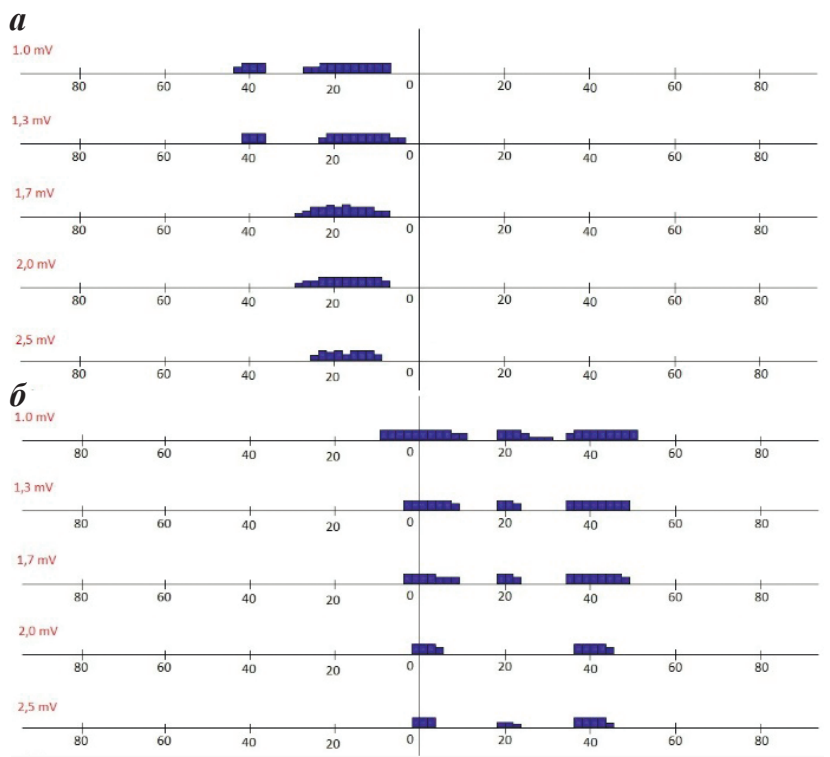

Рис. 1. Демонстрация экрана настройки чувствительности на LS-электроде с нормальной (a) и фрагментированной (б) морфологией сигнала.
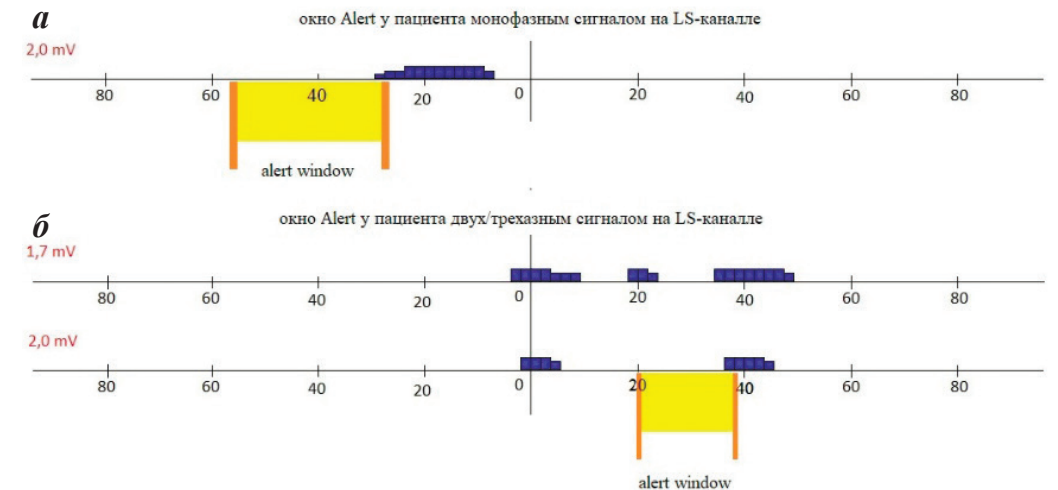

Pис. 2. Окно настройки интервала «Alert window» на LS-электроде при монофазном (а) и при фрагментированном сигнале (б). 
Из трех пациентов с исходным IV ФК NYHА, двое показали умеренное улучшение самочувствия, увеличение дистанции ТШХ и снижение уровня NTproBNP, сопоставимые с положительной динамикой у пациентов с менее выраженными явлениями недостаточности.

Следует отметить, что семи пациентам имплантировались устройства Optimizer IV, для адекватной работы которых требуется наличие синусового ритма. У одного из пациентов на 6-месячном плановом визите был выявлен пароксизм фибрилляции предсердий (восстановление ритма было невозможно ввиду тромбоза ушка левого предсердия), в связи с чем процент работы устройства составил менее $20 \%$ от должного.

Еще одной причиной значимого снижения эффективной работы устройств явилась частая желудочковая экстрасистолия (у одного из наблюдаемых нами пациентов до $17 \%$ от общего числа QRS комплексов). Контроль и коррекция работы устройства выполнялись каждые 2 месяца, на этом фоне процент эффективной работы оставался на уровне 70\%. Эти пациенты отмечали улучшение самочувствия и переносимости физической активности до тех пор, пока устройство адекватно работало, однако по данным ЭхоКГ положительной динамики не отмечалось. У остальных пациентов, завершивших 12-месячное наблюдение процент эффективной работы оставался более 90\%, нежелательных явлений или осложнений МСС-терапии у этих не отмечалось.

\section{ОБСУЖДЕНИЕ ПОЛУЧЕННЫХ РЕЗУЛЬТАТОВ}

По результатам анализа наших данных MCC-терапия была ассоциирована с улучшением толерантности к физическим нагрузкам у пациентов старшей возрастной группы: у этих пациентов отмечалось достоверное увеличение дистанции ТШХ. Положительная динамика наблюдалась также в отношении показателей ФВЛЖ и NTproBNP, хотя эти различия

таблица 2.

Динамика объемных и линейных размеров камер сердца в ходе наблюдения по данным трансторакальной эхокардиографии *

\begin{tabular}{|l|c|c|c|}
\hline & Исходно & Через 12 месяцев & $\mathrm{p}$ \\
\hline ПЗРЛП, мм & $47[42 ; 59]$ & $47[39 ; 56]$ & 0,62 \\
\hline КДР, мм & $63[33 ; 75]$ & $63,0[60 ; 65]$ & 0,38 \\
\hline КСР, мм & $52[50 ; 55]$ & $50[47 ; 50]$ & 0,5 \\
\hline КДО, мл & $208[173 ; 270]$ & $200[177 ; 250]$ & 0,83 \\
\hline КСО, мл & $133[113 ; 150]$ & $133[105 ; 150]$ & 0,67 \\
\hline ФВ ЛЖ, \% & $34[31 ; 38]$ & $40[32 ; 40]$ & 0,2 \\
\hline
\end{tabular}

Примечание: * - данные представлены как медиана и интерквартильный размах, ПЗРЛП - переднезадний размер левого предсердия, КДО - конечный диастолический объем левого желудочка, КДР - конечный диастолический размер левого желудочка, КСО - конечный систолический объем левого желудочка, КСР конечный систолический размер левого желудочка, ФВ ЛЖ - фракция выброса левого желудочка (по методу Simpson) не достигли статически значимой величины, что вероятно связано с относительно коротким сроком наблюдения и малочисленностью выборки. Косвенным подтверждением нашего предположения можно считать данные исследования S.D.Anker et al [8], в котором изучались выживаемость, количество госпитализаций, ЭхоКГ данные и качество жизни у более молодых пациентов $(n=140)$ после первичной имплантации устройств МСС-терапии со сроком наблюдения три года. Авторы показали, что МСС-терапия ассоциировалась с улучшением качества жизни и достоверным увеличением ФВЛЖ, а также снижением количества госпитализаций. С другой стороны важно помнить, что наличие коморбидной патологии и пожилой возраст пациентов с $\mathrm{CH}$, независимо ухудшают прогноз по сравнению с более молодой популяцией: F.Gustafsson et al [9], изучив влияние возраста на сердечно-сосудистую смертность у 5548 пациентов со $\mathrm{CH}$, показали, что за каждое десятилетие риск сердечно-сосудистой смерти увеличивается примерно на треть у пациентов с систолической дисфункцией ЛЖ (отношение рисков 1,29, (95\% ДИ 1,19-1,39)), и практически удваивается у пациентов с СН и сохранной ФВЛЖ (отношение рисков 1,57 , (95\% ДИ 1,43-1,72))[9].

МCC-терапия является опцией для тех пациентов с $\mathrm{CH}$, у которых, несмотря на оптимальную медикаментозную терапию и реабилитацию, сохраняются симптомы недостаточности кровообращения, что также сопряжено с ухудшением прогноза. У 65\% пациентов с CH регистрируется узкий QRS комплекс на ЭКГ, что исключает возможность применения СРТ [10]. Другой контингент пациентов, нуждающихся в дополнительной медицинской помощи по поводу $\mathrm{CH}$, составляют те пациенты, которые не ответили на СРТ. J.Kuschyk et al [11], оценив эффективность МСС-терапии у 17 пациентов, не ответивших на СРТ, отметили достоверное увеличение переносимости физических нагрузок и качества жизни уже на плановом визите через 6 месяцев [11]. В нашем исследовании одному пациенту был имплантирован Optimizer smart в связи с неэффективностью СРТ. Он перенес имплантацию устройства без особенностей и в дальнейшем показал небольшое, но стабильное улучшение в виде увеличения дистанции ТШХ и уменьшения симптомов застойной недостаточности.

В настоящий момент понимания есть ли разница в эффективности МСС у пациентов с различным генезом СН нет, за исключением единичных описаний так называемых супер-респондеров у пациентов с дилатационной кардиомиопатией [12]. Малое количество пациентов с неишемической кардиопатией может быть ограничением нашего наблюдения.

Наличие коморбидного фона у престарелых пациентов вызывало опасения, что имплантация устройства могла быть сопряжена с увеличением рисков операционных осложнений. Об этом свидетельствуют данные наблюдательного исследования со включением 25000 пациентов и сроком наблюдения 5 лет. Авторы показали, что пожилой возраст и наличие коморбидной патологии являются независимыми предикторами 
внутрибольничной летальности [13]. Однако интраоперационных и отсроченных осложнений, связанных с имплантацией устройства МСС-терапии, в нашем исследовании не наблюдалось, что, вероятно связано с четким соблюдением всех рекомендаций имплантации и программирования устройств. Обобщая наши наблюдения и данные литературы, можно заключить, что применение МСС-терапии у пациентов старше 65 лет сопряжена с улучшением переносимости физических нагрузок.

\section{Ограничения исследования}

Отсутствие контрольной группы из более молодых пациентов, маленький объем выборки, относи- тельно короткий срок наблюдения являются ограничениями этого исследования.

\section{ЗАКЛЮЧЕНИЕ}

Применение МСС-терапии у пациентов старше 65 лет с сердечной недостаточностью со сниженной фракцией выброса является безопасным и эффективным методом в качестве дополнительной опции к оптимальной медикаментозной терапии для снижения выраженности симптомов СН. Нужны новые исследования на большей популяции и с более длительным периодом наблюдения для ответа на вопрос улучшает ли МСС-терапия ФВЛЖ или прогноз.

\section{ЛИТЕРАТУРА}

1. Imai M, Rastogi S, Gupta RC, et al. Therapy With Cardiac Contractility Modulation Electrical Signals Improves Left Ventricular Function and Remodeling in Dogs With Chronic Heart Failure. J Am Coll Cardiol. 2007;49(21): 2120-8. https://doi.org/10.1016/j.jacc.2006.10.082.

2. Butter C, Rastogi S, Minden HH, et al. Cardiac Contractility Modulation Electrical Signals Improve Myocardial Gene Expression in Patients With Heart Failure. J Am Coll Cardiol. 2008;51(18): 1784-9. https://doi.org/10.1016/j. jacc.2008.01.036.

3. Kleemann T. Kardiale Kontraktilitätsmodulation: Eine neue Therapieform für herzinsuffiziente Patienten mit schmalem Kammerkomplex? Herz. 2015;40(7): 945-51. https://doi.org/10.1007/s00059-015-4362-8.

4. Borggrefe MM, Lawo T, Butter C, et al. Randomized, double blind study of non-excitatory, cardiac contractility modulation electrical impulses for symptomatic heart failure. Eur Heart J. 2008;29: 1019-28. https://doi. org/10.1016/j.jacc.2008.01.036.

5. Kadish A, Nademanee K, Volosin K, et al. A randomized controlled trial evaluating the safety and efficacy of cardiac contractility modulation in advanced heart failure. Am Heart J. 2011;161(2): 329-337.e2. https://doi. org/10.1016/j.ahj.2010.10.025.

6. Abraham WT, Kuck KH, Goldsmith RL, et al. A Randomized Controlled Trial to Evaluate the Safety and Efficacy of Cardiac Contractility Modulation. JACC Hear Fail. 2018;6(10): 874-83. https://doi.org/10.1016/j. ahj.2010.10.025.

7. Nägele H, Behrens S, Eisermann C. Cardiac contractility modulation in non-responders to cardiac resynchroniza- tion therapy. JACC: Heart Failure. 2008;10(12): 1375-80. https://doi.org/10.1016/j.jchf.2018.04.010.

8. Anker SD, Borggrefe M, Neuser H, et al. Cardiac contractility modulation improves long-term survival and hospitalizations in heart failure with reduced ejection fraction. Eur J Heart Fail. 2019;21(9): 1103-13. https://doi. org/10.1002/ejhf.1374.

9. Gustafsson F, Torppedersen C, Seibak M, et al. Effect of age on short and long-term mortality in patients admitted to hospital with congestive heart failure. Eur Heart J. 2004;25(19): 1711-7. https://doi.org/10.1016/j. ehj.2004.07.007.

10. Lund LH, Jurga J, Edner M, et al. Prevalence, correlates, and prognostic significance of QRS prolongation in heart failure with reduced and preserved ejection fraction. Eur Heart J. 2013;34(7): 529-39. https://doi.org/10.1093/ eurheartj/ehs305.

11. Kuschyk J, Nägele H, Heinz-Kuck K, et al. Cardiac contractility modulation treatment in patients with symptomatic heart failure despite optimal medical therapy and cardiac resynchronization therapy (CRT). Int $J$ Cardiol. 2019;277: 173-7. https://doi.org/10.1016/j.ijcard.2018.10.086.

12. Al-Ghamdi B, Shafquat A, Mallawi Y. Cardiac contractility modulation therapy: Are there superresponders? Hear Case Reports. 2017;3(4): 229-32. https://doi. org/10.1016/j.hrcr.2017.02.004.

13. Swindle JP, Rich MW, McCann P, et al. Implantable cardiac device procedures in older patients: Use and in-hospital outcomes. Arch Intern Med. 2010;170(7): 6317. https://doi.org/10.1001/archinternmed.2010.30. 